\title{
Corrosion Cracks Nucleation By Deformation-Induced Passivity Breakdown
}

\author{
Andrey P. Jivkov ${ }^{*}$ and Per Ståhle \\ Solid Mechanics, Malmö University, 20506 Malmö, Sweden \\ email: andrey.jivkov@ts.mah.se,per.stahle@ts.mah.se
}

\begin{abstract}
A model for corrosion crack nucleation and growth is presented, where the corrosion forms the geometry of the crack tip, thus creating the conditions for strain concentration. The interaction between electrochemical processes and the deformation of the crack tip region is incorporated in a continuum mechanical theory. No crack growth criterion is used. The formation of a crack from a surface depression via a pit is studied. Low frequency cyclic load is considered. At the end of a load cycle a metal oxide compound is growing on the crack surface. It is assumed that there is sufficient time for the chemical process to form a protective film that fully covers the crack surface. This temporarily interrupts the corrosion process. During the application of next load cycle the stretch of the surface breaks the protective film. This creates gaps in the film, which allow dissolution of the uncovered metal. The chemical environment of the crack tip is assumed to be constant and unaffected by the changing geometry as the crack is developing. This leads to a linear relationship between strain and corrosion rate, in the sense of removed material per unit of area during each load cycle. The model simulates how pits evolve to become cracks and how cracks then propagate in one continuous process. Mathematical and finite element analyses of stationary cracks with appropriate geometry are involved to explain the behaviour predicted by the model.
\end{abstract}

Keywords: Stress-corrosion cracking; Crack initiation; Crack evolution; Crack tip geometry; Moving boundaries.

\section{INTRODUCTION}

As many as $25 \%$ of all accidents in process industry reported to the Swedish Plant Inspectorate are caused by stress corrosion crack growth $/ 1 /$. The situation is believed to be similar in all industrialised countries.

\footnotetext{
* Corresponding author. Tel: +46-40-6657626; Fax: +46-40-6657135; E-mail: andrey.jivkov@ts.mah.se
} 
What contributes to the severity of the accidents is that they often occur unexpectedly and at loads that may be far below what normally causes crack growth. The reason for this behaviour, unexpected from the mechanical design perspective, is that the dominant mechanism of crack advance in stress corrosion is the loss of material to the environment, a process of electrochemical nature called dissolution. This process is thermodynamically favourable for the majority of structural metals and alloys if a bare surface of these is exposed to various operational environments /2/. Fortunately, another process called passivation starts operating in parallel with the dissolution. During this process, chemical reactions products from the environment, mainly metal oxides and hydroxides formed by dissolved metal atoms, are being adsorbed at the surface, forming a protective film. The thickness of this protective (also called passive) film is typically 1-4 nm, but sufficient to reduce the rate of dissolution several orders of magnitude. Thus, the existence of passive films on the metal surfaces increases the lives of the structural members tremendously. Failures of the protective film at various positions may, however, occur due to changes in electrochemical conditions in the environment, or as a result of mechanical damage. As the film fails in some surface region, the rate of dissolution may increase to the rate at bare surface conditions, resulting in a (comparatively) rapid material loss. The failure of the film due to mechanical loading is the base for a widely used hypothesis for stress corrosion cracking, where the crack advance results from metal dissolution, localised at the surface region experiencing the highest strains $/ 3,4 /$. This is viewed as a mechanism for maintaining the crack-like geometry during corrosion, as the less strained crack walls remain passive, while in the more strained crack tip region the passive film suffers damage and a bare material surface is exposed to the environment. Because the passivation process restores the passive film, the continuing growth would be possible only by continuing film damage. This means that a constant load cannot provide conditions for continuing crack growth, at least not in the absence of some time dependent material processes, e.g. creep. Late experimental reports provide strong support to this observation $/ 5,6 /$. They show that active loading in terms of either monotonically increasing or fatigue load is an essential prerequisite for continuing corrosion cracking. This also supports the localised dissolution hypothesis for the stress corrosion crack advance mechanism.

Experts in the fields of metallurgy and electrochemistry, who use the localised dissolution hypothesis, rely on mechanical models based on elastic or elastic plastic stress fields, where the crack tip is treated as a point /7, 8/. In view of the near tip load distribution this leads to paradoxical results. It must be recognised in this respect that corrosion cracks observed in reality do not have atomically sharp tips, but rather look like long slender notches. Fig. 1 offers two examples, showing that corrosion cracks possess intrinsic length parameters, e.g. the crack width and the radius of curvature at the tip. An early work considering a more realistic geometry such as this treated the crack as an elliptic hole /9/. The stress and strain fields in the crack tip vicinity were deduced assuming a linear elastic material. A simple geometric analysis shows that the rate of surface advance, necessary to maintain the assumed crack shape during propagation, must be proportional to the square root of the surface stress (or strain). The rate of surface advance is identified as the dissolution rate in this case. The result is valid for the assumed shape only and for the close vicinity of the tip. 

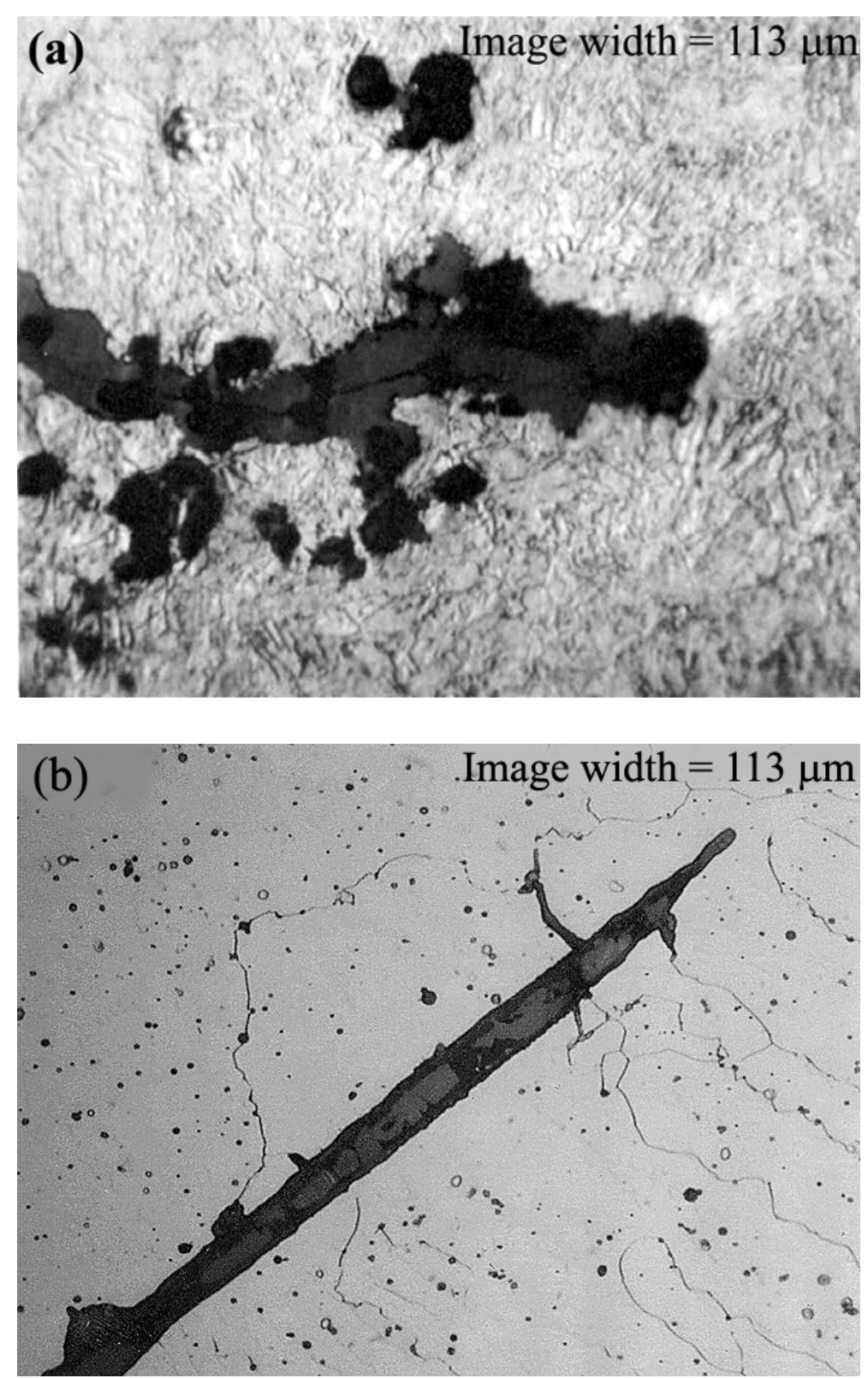

Fig. 1: Corrosion crack examples: (a) in a reactor pressure vessel steel SA 533 C11; (b) in a welding of

The existence of a passive film and the mechanism of its damage by surface straining set a different requirement for the advance rate dependence on strain. A simple model for the passive film damage suggests a linear relation between surface advance rate and strain /10/. This model distinguishes between surface advance rate and dissolution rate, the latter being the electrochemical rate of dissolution for an uncovered 
(bare) metal surface. The model considers only the rupture of the film and assumes the dissolution rate of the bare metal surface to be independent of the surface stress (or strain). This essentially means that the resulting linear relation between rate and strain is a lower bound for a physically more realistic relation, i.e. a dependence of the bare metal dissolution rate on the surface strain would make the surface advance rate dependent on the strain in some non-linear way.

Based on the linear model, a moving boundary problem describing crack surface advance was posed /10/. Numerical solutions of the problem for long plane cracks with parallel walls and various crack tip shapes showed a strong dependence of the growth rate on the initially assumed crack geometry. An important question then would be what in reality determines this geometry? This question might be answered by turning attention to the stage of crack initiation. This stage is worth considering also because it might be the life determining in fatigue corrosion. Pitting has been found to be a major mechanism for crack initiation, and as a rule the largest pits are responsible for the emergence of cracks $/ 11,12 /$. The linear model from /10/, with a corresponding moving boundary problem, was used to study the nucleation of cracks from surface pits in elastic materials $/ 13 /$, and in elastic-plastic materials $/ 14 /$. The results of these works showed that the deformation-film interaction, considered in the model, lead to strain and dissolution localisation, resulting in nucleation of a crack. The entire process of surface evolution, followed in the works, might be split into three stages: crack initiation, linear (or short) crack growth and self-similar growth after crack tip blunting. The present work reviews the results obtained so far on the base of the linear model. It is the geometry of the evolving crack that is of a concern in this study, and the time dimension of the evolution is intentionally avoided. Mathematical analysis and finite element results for stationary cracks with appropriate realistic geometry are also used to explain the observed geometry evolution.

\section{DEFORMATION-INDUCED PASSIVITY BREAKDOWN}

Consider a solid body with a surface in contact with a corrosive environment. A passive film covers the body surface everywhere and compactly. The film is assumed to be composed of metal oxides and therefore brittle under tensile strains. The strain at rupture, denoted by $\varepsilon_{\text {f }}$, is the only additional parameter of the mechanical behaviour of the film. Otherwise, the film is assumed to have the same mechanical properties as the bulk material of the body and its thickness does not enter the model. It is further supposed that when intact, the film prevents any dissolution of the underlying metal. A segment of the film-covered surface is illustrated in Fig. 2, where $d S$ and $d s$ are the lengths of a differential surface element before and after a mechanical deformation, respectively. This deformation is measured by the strain parallel to the surface, given as $\varepsilon=(\mathrm{d} s-\mathrm{d} S) / \mathrm{d} s$. Upon load application during one load cycle the film deforms together with the bulk material. If the strain along the chosen segment reaches the rupture strain, $\varepsilon_{f}$, the film breaks and a number of film fragments separated by gaps of bare metal are created. The film fragments are supposed to fully relax after rupture. This is the situation shown in the figure. The unprotected gaps are exposed to the 
corrosive environment and the metal dissolves, advancing that portion of the surface. The advance occurs normally to the surface. The rate of surface advance at a given point is considered, as explained later, to be a multiplicative composition of a purely mechanical contribution and a contribution from the operating electrochemical processes.

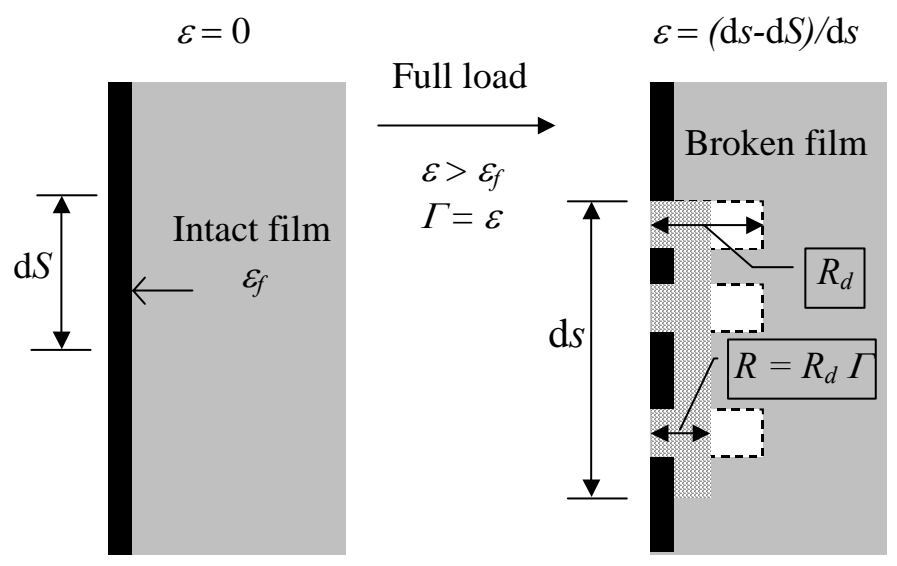

Fig. 2: Illustration of the film damage - dissolution model for surface advance.

The mechanical contribution to the surface advance rate is the degree of passive film damage at the point, denoted by $\Gamma$. This measures how big a portion of the point neighbourhood is "available" for dissolution. In the present model, the degree of damage is determined by "smearing" the advances in the gaps over the deformed length of the differential element. This gives a degree of damage equal to the surface strain $\varepsilon$, as previously defined, if this strain is larger than the rupture strain of the film, $\varepsilon_{f}$. Otherwise, the damage is considered to be zero. Hence, the degree of damage is written as $\Gamma=\varepsilon \theta\left(\varepsilon-\varepsilon_{f}\right)$, where $\theta(x)$ is the Heaviside step function, i.e. $\theta(x)=1$ if $x>0$, and $\theta$. $(x)=0$ otherwise. In the figure, the damage equals the surface strain.

The electrochemical contribution to the surface advance rate depends in principle on the dissolution driving force. This is the electrochemical potential of the solid-environment interface, which is generally a spatial and a temporal variable. The spatial variation throughout the crack geometry may be because of compositional changes in the environment due to mass and charge transport, as well as because of local conditions of straining. In the present model these dependences are neglected and the dissolution rate at a point of bare metal is assumed constant, independent of position and strain. The temporal variation of the driving force, and hence the dissolution rate, is predominantly due to the repassivation process operating simultaneously with the dissolution. For a short time the former restores the film properties of an intact film, i.e. interrupts the active dissolution. The repassivation process causes an exponential decrease of the dissolution rate with time. In order to avoid an explicit time dependence of the results, a simplified integral approach is adopted here, where the load is thought of as applied in cycles of rectangular shape, i.e. quasistatically for each cycle. The cycle period is supposed sufficiently large to allow full restoration of the film before the next cycle is applied. 
With the above assumptions, the advance per load cycle in every gap formed along the film-covered surface is constant, denoted by $R_{d}$ in Fig. 2. In the considered case of rectangular cycles, this constant should be obtained by integration of a given dissolution rate variation over the duration of one cycle. In such case, the surface advance at a point per load cycle is written as

$$
R=R_{d} \Gamma=R_{d} \varepsilon \theta\left(\varepsilon-\varepsilon_{f}\right),
$$

which is also depicted in the figure and is the form used in the following sections. The degree of damage in (1) is the one that is maintained at the point during the peak of the cycle. If cycles of general shape are to be considered, the pre-integration that would give the surface advance per cycle in a form similar to (1) is more elaborative. It requires a convolution of the dissolution rate variation with the rate of straining, or passive film damaging. In such a case, the parameters on the right-hand side of (1) would represent averaged values of the dissolution rate and strain over one load cycle. Considering a particular dissolution rate variation due to repassivation and a more general than rectangular load cycles, however, is not necessary as the emphasis in this work is on the corrosion crack geometry evolution. For this, the quasi-static advance per cycle formulation (1) is sufficient.

\section{THE MOVING BOUNDARY PROBLEMS}
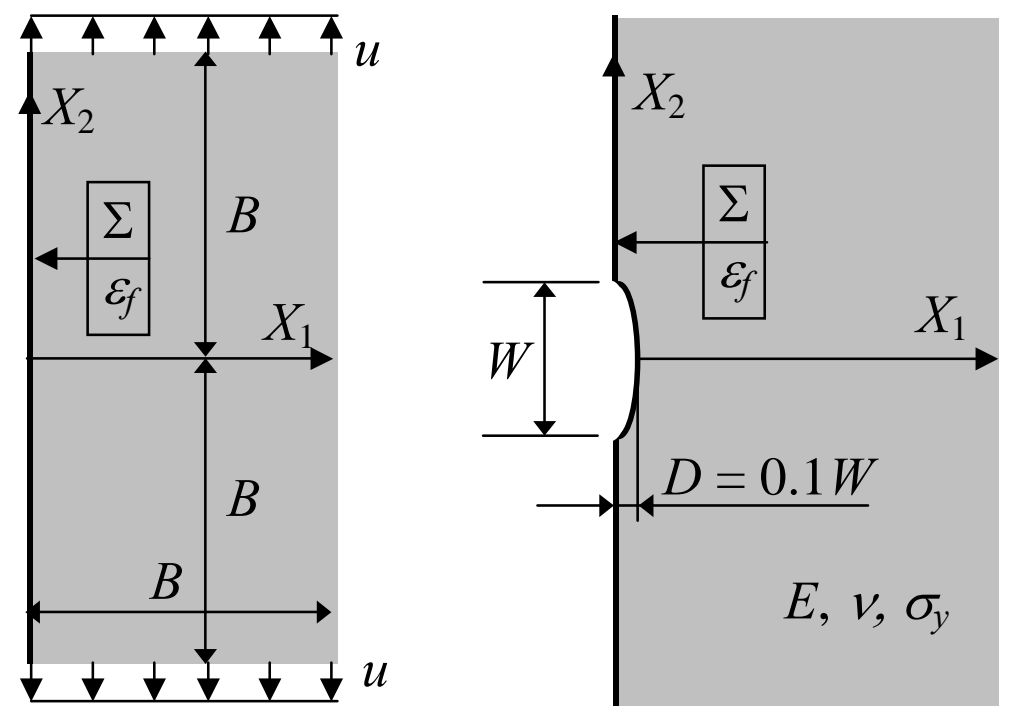

Fig. 3: Geometry of the moving boundary problems studied.

The problems stated in this section share the same initial geometry and boundary conditions, illustrated in Fig. 3, and differ only in the mechanical properties ascribed to the solid material. With a fixed coordinate 
system $\left(X_{1}, X_{2}\right)$, a plane solid body occupying the region $0 \leq X_{1} \leq B$ and $\left|X_{2}\right| \leq B$ is considered. A half elliptic pit, described by $\left(X_{1} / D\right)^{2}+\left(X_{2} / W\right)^{2}=1$ for $X_{1} \geq 0$, is introduced on the surface $X_{1}=0$. The width and depth of this pit are chosen as $W=10^{-3} B$ and $D=0.1 \mathrm{~W}$, respectively, as shown on the left part of Fig. 3. It should be mentioned, that pits of variable depths, $D$, and their influence on crack incubation have been considered in $/ 13 /$, but this issue will not be addressed in the present work. The boundary, which includes the surfaces $X_{1}=$ $0, W \leq\left|X_{2}\right| \leq B$, and the surface of the pit, is denoted by $\Sigma$. This boundary is assumed to be in contact with a corrosive environment and to be initially covered by a passive film. The rupture strain of the passive film is taken to be $\varepsilon_{f}=0.001$. The material of the body is chosen to be either linear elastic or elastic-perfectly plastic, characterised by Young's modulus $E=206 \mathrm{GPa}$, Poisson's ratio $v=0.3$, and for plastic materials by the yield strength $\sigma_{y}$.

The body is considered to be in plane strain. The components of the boundary displacement and traction vectors are denoted by $U_{1}$ and $U_{2}$, and $T_{1}$ and $T_{2}$, respectively. The components of the surface advance per load cycle due to material loss and of the current outward unit normal to the boundary are denoted by $R_{1}$ and $R_{2}$, and $N_{1}$ and $N_{2}$, respectively. The boundary conditions for one load cycle are then given as follows:

$$
\begin{aligned}
& \mid \begin{array}{l}
T_{1}=0 \\
U_{2}=-u
\end{array}, \quad \text { at } 0 \leq X_{1} \leq B \text { and } X_{2}=-B, \\
& T_{1}=T_{2}=0, \quad \text { at } X_{1}=B \text { and }\left|X_{2}\right| \leq B, \\
& \mid \begin{array}{l}
T_{1}=0 \\
U_{2}=u
\end{array}, \text { at } 0 \leq X_{1} \leq B \text { and } X_{2}=B, \\
& \mid \begin{array}{l}
T_{1}=T_{2}=0 \\
R_{1}=-R N_{1} \\
R_{2}=-R N_{2}
\end{array}, \text { at } \Sigma
\end{aligned}
$$

where $u$ is the applied peak displacement during the cycle, and $R$ is the advance per cycle calculated via (1). The peak displacement is chosen as $u=\varepsilon_{f} B$, which ensures a constant strain field in a rectangular body without surface flaws and is exactly on the threshold to break the protective film of the flat surface.

The described above geometry, material and boundary conditions constitute a moving boundary value problem (MBVP). This can be seen from the excess of boundary conditions along $\Sigma$, eqn (2d), which compels the boundary to evolve in a way that the redistributed stresses are maintained in an instantaneous equilibrium with the applied loads. In the present context, each load cycle is understood as a new boundary value problem, with geometry determined by the entire past history of the evolving boundary. The equilibrium solution for the new problem defines the present evolution. This work uses a numerical solution for the MBVP, based on a problem split into equilibrium and evolution sub-problems over each load cycle. 
The equilibrium sub-problem is solved using the commercial finite element analysis program ABAQUS /15/. Constant strain triangular finite elements are used in the analysis. The surface strains, $\varepsilon$, obtained at equilibrium, provide the advance of the corroding surface $\Sigma$ via eqn (1). The evolution sub-problem is solved using an in-house procedure for surface tracking and geometry re-meshing. The evolving part of the surface is described using a cubic B-spline. The spline is reshaped after each load cycle by displacing the surface nodes and then re-meshed to accurately describe the new shape. The spline re-meshing process is based on a length criterion - minimum edge size, $e_{\text {min }}$; and on a curvature criterion - maximum angle between two consecutive edges, $\gamma_{\max }$. A thorough description of the re-meshing process may be found in /16/. The evolved body geometry requires re-meshing of the interior, which is completed with a Delauney-type triangulation procedure. Thus the current geometry is changed and the finite element formulation of the boundary value problem for the next cycle is prepared. The residual stresses and plastic strains, obtained after a load cycle closing, have been neglected when defining the boundary value problem for the next cycle.

\section{MOVING BOUNDARY RESULTS}

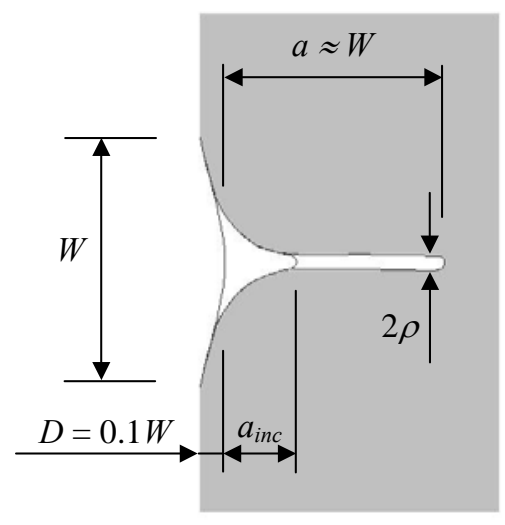

Fig. 4: Evolution of a corrosion crack geometry.

This section reviews some of the results from /13, 14/ and /16/, as they are needed in the following sections of the work. The results presented, are for the particular choice of surface re-meshing parameters $e_{\min }$ $=5 \times 10^{-3} \mathrm{~W}, \tan \left(\gamma_{\max }\right)=0.15$. A normalised load factor, $\sigma_{\infty} / \sigma_{y}$, is introduced, where for the given boundary conditions the remotely applied stress is $\sigma_{\infty}=\varepsilon_{f} E /\left(1-v^{2}\right)$. A number of yield strengths, representing the range from elastic to very soft plastic materials, namely $0 \leq \sigma_{\infty} / \sigma_{y} \leq 0.5$, have been considered. Note, that for edge cracks the load interval $\sigma_{\infty} / \sigma_{y}<0.32$ defines small scale yielding (SSY) conditions according to an ASTM convention. The solutions of the corresponding moving boundary problems show that the surface of the initial pit evolves towards a crack-like shape and then grows as corrosion crack in the sense of deep 
slender notch. The surface evolution before becoming a crack is called incubation and this descriptive definition should be accepted until a more precise one is given in the next paragraph. An illustration of such evolution is given in Fig. 4, where some results for the particular load factor $\sigma_{\infty} / \sigma_{y}=0.15$ are collected in one. These are the profiles of the initial pit, the surface at crack incubation, and the crack at extension $W$. The crack extension, denoted by $a$, is measured from the initial pit surface to the current position of the crack tip, which with reference to the coordinate system in Fig. 3 is the point of largest $X_{1}$-coordinate along $\Sigma$. The incubation crack length is $a_{i n c}$. The crack width in the tip region, denoted by $2 \rho$, is defined as the distance between the points where $45^{\circ}$ lines running back from the crack tip intercept the crack faces.

The crack growth rate, $\mathrm{d} a$, is identified as the advance per load cycle of the crack tip point normalised with the electrochemical advance, i.e. $\mathrm{d} a=\left(R / R_{\mathrm{d}}\right)_{t i p}=\varepsilon_{t i p}$. Fig. 5 shows the crack growth rate variation as a function of the square root of crack extension, normalised with the initial pit width $W$, for three chosen values of the yield strength, $\sigma_{\infty} / \sigma_{y}=0, \sigma_{\infty} / \sigma_{y}=0.15, \sigma_{\infty} / \sigma_{y}=0.30$. Note, that the first value represents an elastic material.

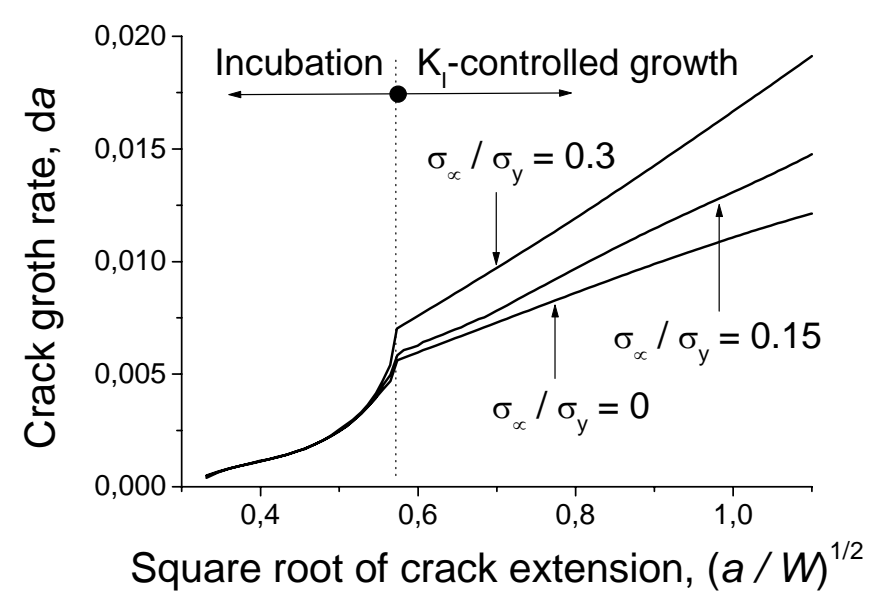

Fig. 5: Crack growth rate development.

As presented, the graph illustrates a clear separation between two stages of evolution that may also be distinguished on the fracture mechanical base. The stress intensity factor, $K_{I}$, for a sharp crack of length $a$, emerging from a surface pit with dimensional notations of Fig. 3, is:

$$
K_{I}=\sigma_{\infty} \sqrt{\pi a} f(a, D, W),
$$

where $f(a, D, W)$ is a geometry factor, that may be found in e.g. $/ 17 /$. The elastic solution for a deep slender notch with circular tip region of radius $\rho$ (see /17/), provides an approximate value for the maximum strain at the tip: 


$$
\varepsilon_{t i p}=\frac{1-v^{2}}{E} \frac{2 K_{I}}{\sqrt{\pi \rho}},
$$

where $K_{I}$ is determined via (3). On the basis of this proportionality between $K_{I}$ and the strain at the tip one concludes that there is a $K_{\Gamma}$-controlled zone surrounding the notch tip and the notch could be considered as a crack at sufficiently large length scale. In the considered case of corrosion crack $\varepsilon_{t i p}=\mathrm{d} a$, and a linear relation between crack growth rate and the square root of crack extension in the results would also mean existence of a $K_{\Gamma}$-controlled zone surrounding the crack tip. An approximately linear relation between these two parameters is seen in the second stage of evolution in Fig. 5 for all materials shown. Therefore, the latter is called stage of $K_{\Gamma}$-controlled growth. The preceding stage is naturally called incubation. It is characterised by strain and dissolution localisation that lead to crack growth acceleration and eventual crack formation at the offset. So, a more precise definition for the incubation instance would be the onset of (approximately) linear relation between crack growth rate and square root of crack extension. Fig. 5 also shows that the crack extension at incubation, $a_{i n c}$, is independent of the initial geometry and material yield strength, while the crack acceleration during propagation is clearly dependent on the material. Before turning attention to the latter observation, it is important to demonstrate the behaviour of the crack width. Fig. 6 shows the development of crack half-width, $\rho$, for the same materials as in Fig. 5. Because the graphs of the three evolutions are almost coinciding, they are given with solid, dashed and dotted lines, for $\sigma_{\infty} / \sigma_{y}=0, \sigma_{\infty} / \sigma_{y}=$ 0.15 and $\sigma_{\infty} / \sigma_{y}=0.30$, respectively. The somewhat fuzzy behaviour of the crack width comes from the inaccuracy of the numerical determination of this parameter and has to be accepted. It must be noted, that during the incubation stage, the crack width is measured in the way described for propagating crack, unless the $45^{\circ}$ lines running back from the tip do not yet intercept the pit faces. In this case the crack width, $2 \rho$, is set to the pit width, $W$. Hence, the curves in the stage of incubation start from the value $0.5 \mathrm{~W}$, but are not entirely shown for convenience.

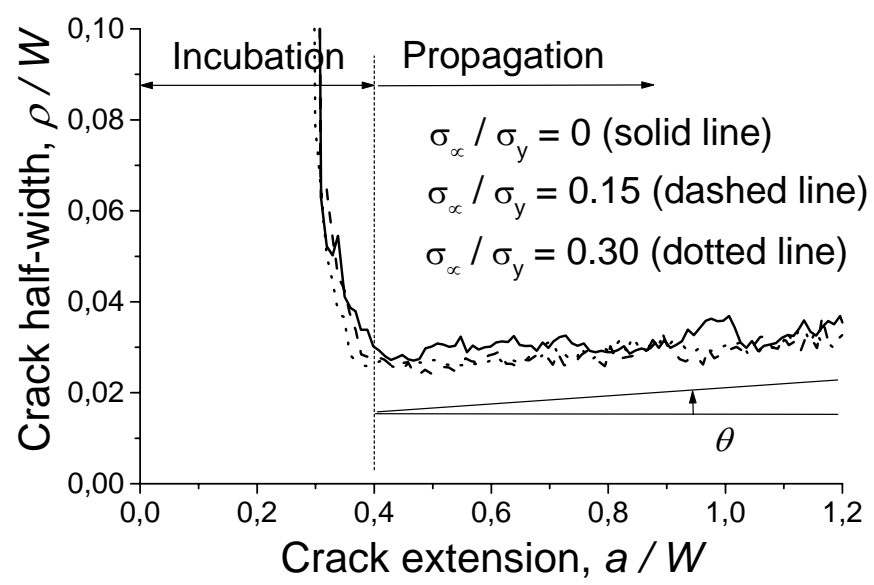

Fig. 6: Crack half-width development. 
In spite of the numerical uncertainty introduced in Fig. 6, some solid observations could be made from the figure. Firstly, Fig. 6 shows again the independence of the incubation crack extension from the material yield strength. Secondly, the crack width attained at incubation is also found to be independent of the material yield strength and of the initial pit geometry. This is actually the instance where the evolving pit surface attains the smallest curvature at the tip during the entire evolution. Thirdly, the crack width during the propagation stage starts increasing slowly and nearly linearly. The rate of this increase is almost independent of the material properties, and is measured by the angle $\theta$ that the crack face makes with the direction of propagation. As discussed in the next sections, the crack width increase is a consequence of the assumed model for surface advance, eqn (1). It is characterised by corrosive blunting of the tip, which continues until the crack attains a shape allowing a steady-state growth, i.e. at constant rate $\mathrm{d} a$. During this growth the geometry of the crack is maintained self-similar, in such a way that all strains along the surface in the tip region are kept constant while that region expands as a void /13, 14/.

The crack growth rate attained at incubation and its development during propagation shows strong dependence on the material yield strength, Fig. 5. At the same time the crack width is independent of the material properties, Fig. 6. This suggests that shape changes of the crack tip region occur. To demonstrate the extent of these changes an appropriate scaling factor based on the elastic solution for a long slender notch is used. Using eqns (3) and (4) with $\sigma_{\infty}=\varepsilon_{f} E /\left(1-v^{2}\right)$, one can define a dimensionless shape factor by:

$$
\phi=\alpha \frac{\mathrm{d} a}{\varepsilon_{f}} \sqrt{\frac{\rho}{a}},
$$

where $\alpha=1 /(2 f)$ is a factor depending on the geometry through $f(a, D, W)$. For an edge crack, emerging from a pit with dimensions of Fig. 3, $\alpha \approx 0.4458 / 17 /$. Note from eqn (4), that $\phi=1$ if the notch has exactly circular tip shape and the material is elastic. Note also that for a given yield strength, $\phi$ is proportional to the slope of the corresponding line in Fig. 4 representing crack propagation stage.

The dependence of the shape factor on the yield strength is presented in Fig. 7. The graph shows that the material yield strength is a parameter controlling the shape of the formed crack tip region. For elastic and very hard plastic materials, this region may be thought of as circular, and changes towards probably an ellipse with decreasing yield strength. The precise shapes, however, cannot be determined only on the base of the crack tip strain. An analysis of the surface strain distribution over the crack faces is needed. 


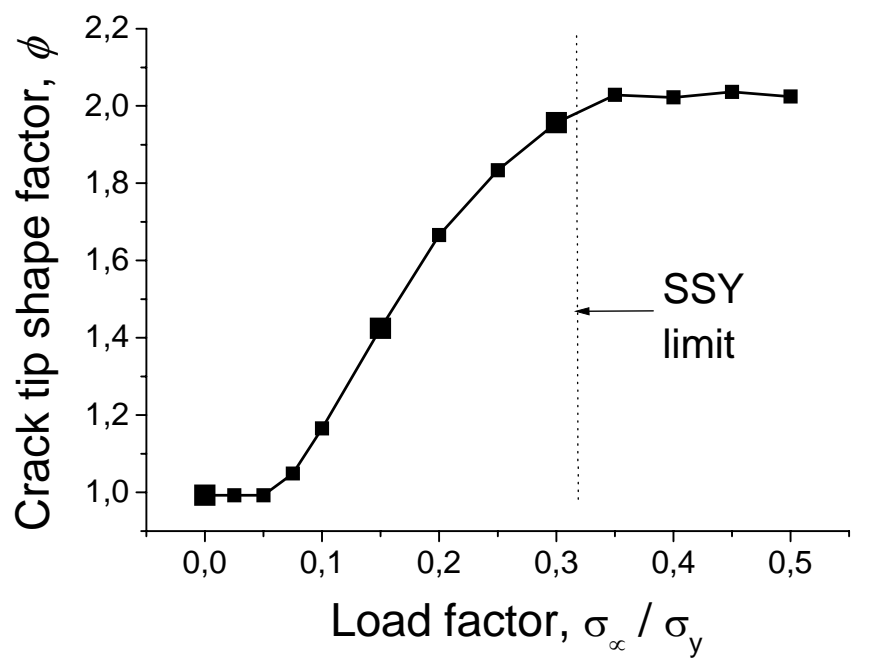

Fig. 7: Shape factor vs. yield strength.

\section{ANALYSIS OF A STEADY STATE CORROSION CRACK}

The maximum strain at the tip of a long slender notch with circular tip, eqn (4), is determined from the analytical solution for the strain field surrounding the tip of an elliptic hole /9/. This analytical solution is only approximately valid for the close vicinity of the tip, where the differences between the ellipse and the osculating circle are small in terms of curvature. Here the surface strain distribution along the entire curved part of the tip and not only the foremost point is essential. Therefore, numerical solutions for a long slender notch with half circular tip have been performed to demonstrate an interpretation of the results from the previous section.

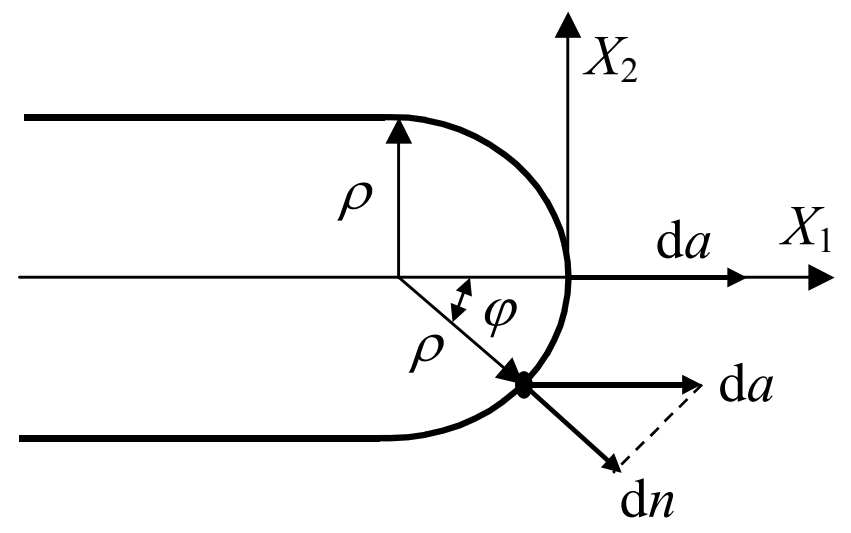

Fig. 8: Long plane notch with circular tip. 
Finite element analysis is used. A large body with a long crack of parallel walls and a circular tip region is considered. A standard boundary layer formulation of the problem is utilised. The layer is a circular region surrounding the crack tip and centred at the tip. The radius of the tip is $\rho$, while the radius of the boundary layer is taken to be $r=100 \rho$. This is considered sufficient for the $K_{1}$-terms in the expansions of stress, strain and displacement fields to dominate at the circular boundary, $r$, of the layer. The geometry of the crack tip region is shown in Fig. 8, together with the coordinate system that will be used. Before continuing with the problem formulation and solution, the conditions of strain necessary to maintain the geometry of Fig. 8 selfsimilar during propagation, i.e. the conditions for steady-state growth parallel to $X_{1}$, are considered. Geometrically, steady-state growth imply that the $X_{1}$-component of the advance of every point along the crack surface should be the same and equal to the advance of the crack tip, denoted as before by d $a$. For an arbitrary surface point $n$ on the circular part of the tip, this means that the necessary advance, $\mathrm{d} n$, normal to the surface is given by

$$
\mathrm{d} n=\mathrm{d} a \cos \varphi=\mathrm{d} a\left(1+\frac{X_{1}}{\rho}\right)
$$

using the notations of Fig. 8. On the upper and lower parallel straight crack surfaces the advance disappears. The linear model given by eqn (1) yields $\mathrm{d} n / \mathrm{d} a=\varepsilon / \varepsilon_{t i p}$ where $\varepsilon$ is the strain at the point $n$. Hence, eqns (1) and (6) require a distribution of surface strains that is given by

$$
\varepsilon=\varepsilon_{t i p}\left(1+\frac{X_{1}}{\rho}\right)
$$

Since the profiles of longer cracks resemble the geometry depicted in Fig. 8, the aim here is to examine how well the strain field produced around the notch of Fig. 8 fulfils eqn. (7). Because the barrier posed by the rupture strain of the passive film, $\varepsilon_{f}$, is a lower limit strain, under which corrosion does not occur, the strain on the straight parts of the crack surfaces must be less than $\varepsilon_{f}$ and equal to $\varepsilon_{f}$ at the point where the straight and the half circular segments are connected, i.e. at $X_{1}=-\rho$. This is a condition for the crack faces to stay parallel.

To find support for the suggested strain distribution (7), finite element calculations have been carried out. The geometry of the boundary layer problem has been already described above. The material of the layer is again assumed to be elastic or elastic-perfectly plastic with properties as defined in the previous section. Plane strain conditions are considered and the load is applied via prescribed displacements along the circular boundary of the layer. Only the $K_{1}$-terms in the expansions for the displacements are taken. The analysis is performed using six-node triangular finite elements with quadratic interpolation in ABAQUS. 


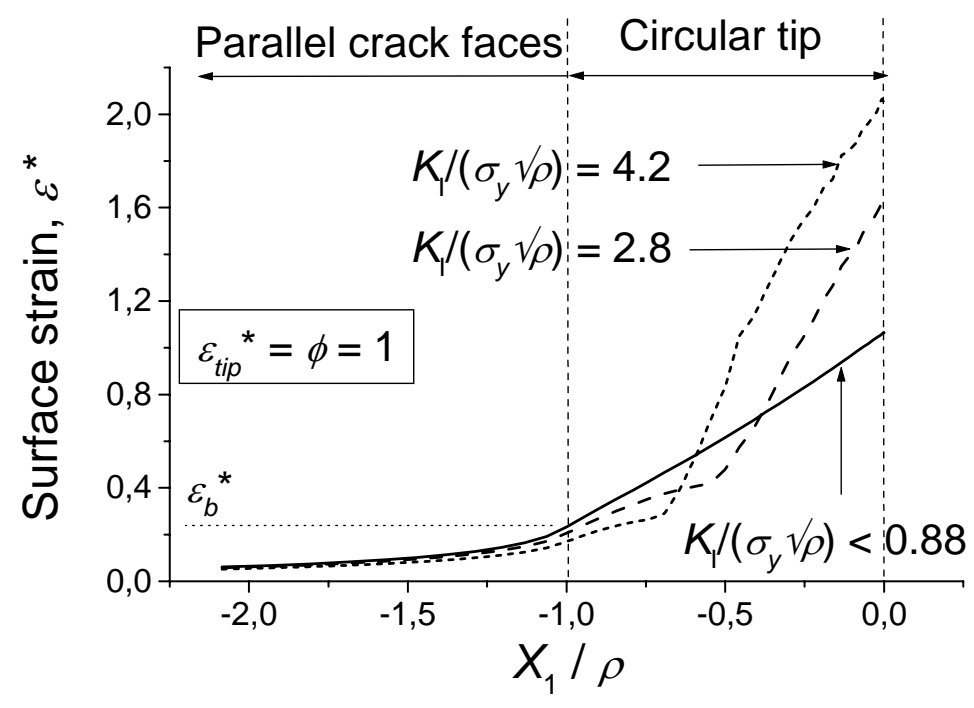

Fig. 9: Surface strain distributions for stationary corrosion crack.

The surface strain distribution for three cases $K_{\mathrm{I}} /\left(\sigma_{y} \sqrt{\rho}\right) \leq 0.88, K_{1} /\left(\sigma_{y} \sqrt{\rho}\right)=2.8$ and $K_{1} /\left(\sigma_{y} \sqrt{\rho}\right)=4.2$ is shown in Fig. 9 (these correspond to $\sigma_{\infty} / \sigma_{y}=0, \sigma_{\infty} / \sigma_{y}=0.10$ and $\sigma_{\infty} / \sigma_{y}=0.15$, respectively). The distribution is given as a function of the $X_{1}$-coordinate, normalised width the crack width $\rho$. For convenience in comparison, the surface strain is also normalised using the form and notations of eqn. (5). Thus, the graph shows the variable:

$$
\varepsilon^{*}=\frac{\varepsilon E \sqrt{\pi \rho}}{2\left(1-v^{2}\right) K_{\mathrm{I}}},
$$

In the figure, $\varepsilon_{b}^{*}$ denotes the normalised strain at the border between the flat crack face and the circular tip region. Note that the normalised strain at the tip in elastic material should be unity, $\varepsilon_{\text {tip }}^{*}=\phi=1$. There is a small discrepancy between this value and the numerical result in Fig. 9, because eqn. (4) is a result of a solution for an elliptic hole, which approximates the actual strain at the tip for the considered long slender notch with parallel walls.

Fig. 9 shows a (nearly) linear distribution of surface strains for the case of elastic material, which supports the observation that the steady-state solution has a shape close to the one given in Fig. 8. The threshold strain is $\varepsilon_{b}^{*}=0.2334$ read from Fig. 9. Now, we are provided with the necessary prerequisites to calculate the crack half width, $\rho$. The conclusion, considering eqn. (8), is that 


$$
\rho=0.057\left(\frac{K_{\mathrm{I}}}{\varepsilon_{f} E}\right)^{2},
$$

The crack width increases with increasing $K_{\mathrm{I}}$, e.g. for an edge crack where $K_{\mathrm{I}}$ is proportional to $\sqrt{ } a$, the crack width becomes proportional to $a$, meaning that the straight parts of the upper and lower crack surfaces form an angle. It becomes obvious, that a small value of remote load, as compared with $\varepsilon_{f} E$, maintaining a small angle between the crack surfaces is a prerequisite for the present steady-state analysis.

Another noteworthy detail is that the relation between the strain at the tip and the threshold strain is maintained at $\varepsilon_{t i p}^{*} / \varepsilon_{b}^{*}=4.56$ for the lowest load. Hence, the crack growth rate at steady-state is given by

$$
\mathrm{d} a=\varepsilon_{t i p}=\left(\varepsilon_{t i p}^{*} / \varepsilon_{b}^{*}\right) \varepsilon_{f}=4.56 \varepsilon_{f},
$$

Opposed to crack growth in general, the growth rate is independent of remote load as long as the load is sufficiently small. Figure 9 shows that plasticity leads to a distribution of surface strains that increases the corrosion rate in the vicinity of the tip and decreases the rate close to the border as compared to elastic material. The analysis does not allow a definite description of the surface shape, judging from strain distribution alone. However, a comparison based on the case with the largest scale of plasticity, $K_{\mathrm{I}} /\left(\sigma_{y} \sqrt{ } \rho\right)=$ 4.2, of the maximum strain at the tip shown in Fig. $9, \varepsilon_{\text {tip }}^{*}=2.07$, and the one shown in Fig. $6, \varepsilon_{\text {tip }}^{*}=1.42$, suggests a trend. Seemingly because the solution for circular tip geometry provides a larger strain at the tip, the growing crack at a higher load has smaller curvature in the tip region, i.e. the corrosion crack is more blunt in plastic material compared to elastic material.

\section{DISCUSSION AND CONCLUSIONS}

The aim of the present work was to review previous and present some new results on nucleation and propagation of fatigue corrosion cracks as well as to examine them with the help of analysis of stationary corrosion cracks. The results presented, are solutions of moving boundary value problems. These arise from the evolutionary character of the corrosion crack initiation and growth as smooth surface advance cased by material loss. The model for surface advance, described in section 2, accounts for three basic processes deformation, dissolution and repassivation - in a simplified way. The deformation causes rupture of an existing passive film, but neither the dissolution rate of the uncovered bare metal nor the rate of repassivation is regarded as deformation dependent. Further, these two rates are supposed to be constant during crack evolution, i.e. the possible transport processes in the external environment, which could produce spatial variations of these rates, are uncared for. The result of these simplifications is the linear relation between 
surface strain and surface advance. Despite some insufficiencies of the physical model suggested, the moving boundary formulation of section 3 proved to be effective in studying the nucleation and propagation of corrosion cracks. The results presented in section 4 show the importance of the deformation-corrosion interaction for strain and dissolution localisation during crack incubation. The end of incubation is marked by the appearance of a $K_{I}$-controlled zone surrounding the crack tip. As examined in section 5, the shape of the formed crack tip is either close to circular for elastic and very hard plastic materials or more complex, but necessarily more blunted for softer plastic materials. This is a consequence of the linear model for surface advance. The subsequent growth under $K_{I}$-control is characterised by almost linear increase of the crack growth rate. The deviation from linearity, expected on theoretical grounds, is due to the increase of the crack width. This increase is a consequence of the advance threshold posed in the model by the rupture strain of the passive film. The crack width is found to depend linearly on the crack extension and this was also characterised in section 5 .

The behaviour predicted on the basis of the linear model is not confirmed categorically by the appearance of corrosion cracks as those in Fig. 1. In both cases one observes an established crack width that has been maintained nearly constant for a long period of growth. There are several physical reasons that have not been taken into account in the model but might affect the real behaviour. For example, in the case of inter-granular corrosion cracking in stainless steels the grain boundary (inter-phase) thickness limits from above the crack width. The Cr-rich grains could be regarded as non-dissolving, while the Cr-depleted inter-phase is susceptible to corrosion. The situation might be described as a tunnel-like area where the crack is allowed to grow. If a crack of width originally smaller than the inter-phase thickness is evolving in the tunnel according to the model prediction, the grains will block the continuous width increase at some point of the evolution. After that, the grains could be regarded as a barrier for surface advance that replaces $\varepsilon_{f}$.

A prospect for further development of the model is to better describe the dissolution rate, $R_{d}$, presently assumed constant. Firstly, the bare surface dissolution rate is altered in a stressed solid. For example, an approximately linear relation between the bare surface dissolution rate and the square root of the logarithmic strain has been reported /18/. Incorporating this relation into the model would make the advance rate dependent on surface strain to the power of approximately 1.5. Note, however, that this step alone would not lead to qualitative change of the corrosion crack behaviour reported here. Secondly, during crack nucleation and propagation the transport processes in the corrosive environment, e.g. diffusion and electro-migration, lead to a distributed chemistry composition in the cavity, see e.g. /19/. This causes variation of the dissolution driving force and hence the dissolution rate along the cavity depth. The difficulty arising here is that the driving force variation depends on the current crack geometry and has to be found as a solution of a separate and more complex moving boundary problem. Nevertheless, this line of future work might be the most fruitful for model improvement. 


\section{ACKNOWLEDGMENTS}

The financial support of the Swedish Centre for Nuclear Technology (SKC) and the Knowledge Foundation (KKS) is highly appreciated.

\section{References}

1. M. Bergman, M. Brickstad and F. Nilsson, A procedure for estimation of pipe break probabilities due to IGSCC. International Journal of Pressure Vessels and Piping, 74(3), pp. 239-248 (1997).

2. P.R. Roberge, Handbook of Corrosion Engineering. McGraw-Hill, New York, 2000.

3. R.N. Parkins, Current understanding of stress-corrosion cracking. Journal of Metals, 44(12), 12-19 (1992).

4. A. Turnbull, Modelling of environment assisted cracking. Corrosion Science, 34(6), 921-960 (1993).

5. K. Kussmaul, D. Blind and V. Läpple, New observations on the crack growth rate of low alloy nuclear grade ferritic steels under constant active load in oxygenated high-temperature water. Nuclear Engineering \& Design, 168(1-3), 53-75 (1997).

6. J. Heldt and H.P. Seifert, Stress corrosion cracking of low-alloy, reactor-pressure-vessel steels in oxygenated, high-temperature water. Nuclear Engineering \& Design, 206(1), 57-89 (2001).

7. F.P. Ford, Quantitative prediction of environmentally assisted cracking. Corrosion, 52(5), 375-395 (1996).

8. G.R. Engelhardt, D.D. Macdonald and M. Urquidi-Macdonald, Development of fast algorithms for estimating stress corrosion crack growth rate. Corrosion Science, 41(12), 2267-2302 (1999).

9. M. Creager and P.C. Paris, Elastic field equations for blunt cracks with reference to stress corrosion cracking. International Journal of Fracture Mechanics, 3, 247-252 (1967).

10. A.P. Jivkov and P. Ståhle, Strain-driven corrosion crack growth - a pilot study of intergranular stress corrosion cracking. Engineering Fracture Mechanics, 69(18), 2095-2111 (2002).

11. Y. Kondo, Prediction of fatigue crack initiation life based on pit growth. Corrosion Science, 45(1), 711 (1989).

12. S.I. Rokhlin, J.-Y. Kim, H. Nagy and B. Zoofan, Effect of pitting corrosion on fatigue crack initiation and fatigue life. Engineering Fracture Mechanics, 62(4/5), 425-444 (1999).

13. A.P. Jivkov, Evolution of fatigue crack corrosion from surface irregularities. Theoretical \& Applied Fracture Mechanics, 40(1), 45-54 (2003).

14. A.P. Jivkov, Surface irregularities as sources for corrosion fatigue. In: Mesomechanics of computation and design of use-specific materials, Sih, G.C., Sakai, S., Panin, V.E. (Eds.), Proc. of the 5th Int. Conf. for Mesomechanics, Tokyo, Japan, pp. 184-191, 2003.

15. ABAQUS User’s Manual, Version 6.3, Hibbitt, Karlsson \& Sorensen Inc., 2002. 
16. A.P. Jivkov, A moving boundary model for fatigue corrosion cracking. In: Moving Boundaries VII, Mammoli, A.A., Brebbia, C.A. (Eds.), Proc. of the $7^{\text {th }}$ Int. Conf. on Computational Modelling of Free and Moving Boundary Problems, Santa Fe, New Mexico, USA, pp. 55-64, 2003.

17. H. Tada, P.C. Paris and G.R. Irwin, The stress analysis of cracks handbook, 3d Ed. ASME Pres: New York, 2000.

18. M.A.V. Devanathan and M.J. Fernando. Kinetics of charge transfer at mechanically strained copper electrodes - I. Anodic dissolution. Electrochim. Acta, 15, 1623-1636 (1970).

19. S.M. Sharland, A mathematical model of the initiation of crevice corrosion in metals, Corros. Sci. 33(2), 183-201 (1992). 